\title{
Calidad de vida del paciente oncológico terminal asistente a una Unidad de Terapia Especializada
}

\author{
Jackelyn Cecilia Salas Pardo ${ }^{1}$
}

\author{
Salas PJC. Calidad de vida del paciente oncológico \\ terminal asistente a una Unidad de Terapia \\ Especializada. Cuid salud, ene-jun 2014; 1(1).
}

\section{RESUMEN}

Introducción: los estudios de calidad de vida en pacientes oncológicos permiten devolver la atención al paciente y a sus necesidades. Objetivo: determinar la calidad de vida del paciente oncológico terminal en la Unidad de Terapia del Dolor y Cuidados Paliativos del Hospital Nacional Guillermo Almenara Irigoyen, Lima/Perú. Metodología: estudio de enfoque cuantitativo, diseño descriptivo de corte transversal, realizado a 51 pacientes con diagnóstico de cáncer terminal. Se utilizó como instrumento un cuestionario y como técnica la entrevista y la visita domiciliaria durante el primer trimestre 2011; se usó la estadística descriptiva. Resultados: los pacientes oncológicos terminales presentaron una calidad de vida media con 41,2\% (21); según las dimensiones de la calidad de vida, la dimensión psicológica fue la más afectada con 47,1\% (24), seguido de la dimensión social con 33,3\% (17) y dimensión funcional con $27,5 \%$ (14). Conclusión: la calidad de vida media predominó entre los pacientes oncológicos; dato que unido a los resultados por dimensiones ofrecen insumos para reflexionar sobre la situación del paciente terminal y del papel que ejercen las Unidades de Cuidados Paliativos en el soporte a la familia.

Palabras clave: cáncer, paciente terminal, cuidados paliativos, enfermería.
Salas PJC. Life quality of terminal oncology patient from a Specialized Therapy Unit. Cuid salud, ene-jun 2014; 1(1).

\begin{abstract}
Introduction: Studies of life quality in oncology patients allows returning the patient care and to their needs. Objective: to determine the life quality of terminal cancer patient at the Unit of Pain Therapy and Palliative Care from "Hospital Nacional Guillermo Almenara Irigoyen”, Lima /Peru. Methodology: a quantitative focus research, descriptive cross-sectional design, performed with 51 terminal cancer diagnosed patients. A questionnaire was used as a tool and interview and home visit as techniques during the first trimester 2011; descriptive statistics were used. Results: terminal cancer patients showed a half-life quality with $41.2 \%$ (21); according to the dimensions of life quality, the psychological dimension was the most affected with $47.1 \%$ (24), followed by the social dimension with $33.3 \%$ (17) and functional dimension with $27.5 \%$ (14). Conclusions: the average of life quality among cancer patients predominated; data that sum with the results by dimensions guides to reflection about situation of the terminal patient and the role of Palliative Care Units in family support.
\end{abstract}

Keywords: cancer, terminal patient, palliative care, nursing.

\footnotetext{
1 Licenciada Enfermera. Egresada de la Escuela de Enfermería Padre Luis Tezza, afiliada a la Universidad Ricardo Palma, LimaPerú.
} 


\section{INTRODUCCIÓN}

En la actualidad, el cáncer es considerado un problema de salud pública a escala mundial, no sólo por sus elevadas tasas de morbilidad y mortalidad, sino por los años de vida perdidos por discapacidad. Mundialmente, los diagnósticos de cáncer más frecuente son: de pulmón [1,8 millones (13,0\% del total)], mama [1,7 millones $(11,9 \%)] \mathrm{y}$ colorectal [1,4 millones (9,7\%)]. Mientras que, entre las causas más comunes de muerte por cáncer destacan: el de pulmón [1,6 millones $(19,4 \%$ del total)], hígado [0,8 millones $(9,1 \%)]$ y estómago $[0,7$ millones $(8,8 \%)]{ }^{1}$

Un informe de la Agencia Internacional de Investigación en Cáncer (IARC), agencia especializada de la OMS, estimó un incremento global en 14,1 millón de nuevos casos de cáncer y 8,2 millones de muertes en el 2012, comparado con 12,7 millones y 7,6 millones, respectivamente, en el 2008. Para el 2025 se estima un incremento sustancial a 19,3 millones de nuevos casos de cáncer, debido al crecimiento demográfico y al envejecimiento de la población; ${ }^{1}$ siendo que más del $70 \%$ de las defunciones correspondería a países en desarrollo cuyos recursos disponibles para la prevención, detección, diagnóstico y tratamiento del cáncer son limitados o inexistentes. ${ }^{2}$

La OMS refiere que el cáncer es la segunda causa de defunciones por enfermedades no trasmisibles (ENT) a nivel mundial. Se prevé que durante los próximos decenios la importancia del cáncer como causa de morbilidad y mortalidad vaya en aumento en todas las regiones del mundo. ${ }^{3}$

El cáncer se ha convertido en la primera causa de muerte en algunos de los países más desarrollados del mundo, superando incluso a las enfermedades cardiovasculares, que tradicionalmente ocupaban este lugar; ${ }^{4}$ situación que ocurrirá por el cambio en los estilos de vida de la población (consumo de tabaco, alcohol, dieta poco saludable, inactividad física), causando estragos en todo el mundo. ${ }^{3}$

Se destaca que la incidencia general de cáncer es menor en América Latina (tasa estandarizada por edad de 163 por 100 000) comparado con Europa (264 por 100 000) o los EEUU (300 por 100 000); sin embargo, la mortalidad aún sigue siendo mayor, debido principalmente a la detección tardía del cáncer, que en parte, está relacionado con el menor acceso a los servicios de salud para su diagnóstico y tratamiento. $^{5}$

En el Perú, la Vigilancia Epidemiológica de Cáncer, periodo 2006-2011, notificó en promedio 18319 casos nuevos por año, variando el número de casos incidentes entre 16671 (año 2006) y 19 461 (año 2011), con un pico de 19455 en el 2008. El Registro Poblacional de Cáncer de Lima Metropolitana permite estimar que el 2011 se diagnosticaron en Lima Metropolitana un total de 34000 casos nuevos. Si a estos datos se incluyen los del Registro de Cáncer Poblacional de Arequipa y los del Registro de Cáncer de Base Poblacional de Trujillo, para el año 2015 se diagnosticarían 46264 casos nuevos de cáncer a nivel nacional. ${ }^{5}$ Considerándose un problema de salud tan común en la sociedad, debido al aumento de su frecuencia y al daño que ocasiona en hombres y mujeres al causar muerte, discapacidad e invalidez prematura, con impacto importante en la economía del país, ya que se pierde casi 900 millones de dólares anuales por muerte prematura y vida con discapacidad. ${ }^{6}$

Como es de conocimiento, el cáncer lleva a la persona a una condición de debilitamiento que conduce a una fase terminal, caracterizada por la fase final de una enfermedad crónica, avanzada e incurable que no responde al tratamiento curativo. Este proceso irreversible de la enfermedad desencadena pérdida de la dignidad, miedo a la muerte, al dolor, crisis y un sufrimiento intenso e incapacitante en el paciente, estado que provoca en muchos de ellos la solicitud de eutanasia y asistencia al suicidio. Ante esta situación de dolor y sufrimiento, el objetivo de la medicina debería de cambiar, anteponiéndose el CUIDADO (que posee un modelo biopsicosocial) ante el ideal de CURAR, medicina dominada por la lógica del "imperativo tecnológico". 7

El cáncer en estado terminal, así como los efectos secundarios del tratamiento oncológico para prolongar el tiempo de vida, afecta de manera sustancial la vida de los pacientes; motivo por el cual surgen los Cuidados Paliativos, en respuesta a las necesidades de los pacientes y familiares, con el objeto de mejorar su calidad de vida y constituir una alternativa tangible a la eutanasia y al suicidio asistido.

En la literatura nacional, existe escasa o nula publicación sobre la calidad de vida en pacientes oncológicos en fase terminal, siendo que el objetivo de este estudio fue determinar la calidad 
de vida del paciente oncológico atendido en la Unidad de Terapia del Dolor y Cuidados Paliativos del Hospital Nacional Guillermo Almenara Irigoyen (HNGAI).

\section{METODOLOGÍA}

Estudio de enfoque cuantitativo, diseño descriptivo de corte transversal realizado en la Unidad de Terapia del Dolor y Cuidados Paliativos del HNGAI/EsSalud, en la ciudad de Lima-Perú; se realizó un muestreo no probabilístico intencional constituido por 51 pacientes oncológicos en fase terminal considerando como criterios de inclusión: diagnóstico de cáncer, con edad $\geq 30$ años de edad, pronóstico de vida $\leq 6$ meses, con familiar acompañante y participación voluntaria. Entre los criterios de exclusión se consideró el estado de agonía, la limitación auditiva, el deterioro cognitivo y ser nuevo en el servicio, estar hospitalizado en el momento del estudio y tener otros diagnósticos sobre agregados.

Para la recolección de datos se utilizó como técnica la visita domiciliaria y la entrevista; como instrumento se usó un cuestionario estructurado que contenía 30 ítems con respuestas dicotómicas, evaluándose 4 dimensiones: funcional, psicológico, social y espiritual. Se usó la Escala de Karnofsky Performance Status para determinar el grado funcional del paciente en la realización de actividades físicas.

El cuestionario fue elaborado por la autora basado en la literatura internacional, siendo sometido a juicio de expertos, obteniéndose un grado de concordancia significativa $(\mathrm{p}=0,02)$; también se realizó la prueba piloto con 12 pacientes, resultados que fueron sometidos a la prueba KR-20 con una confiabilidad de $91 \%$.

Obtenida la aprobación del comité de investigación y ética de la institución (Carta $\mathrm{N}^{\circ}$ 3529-OCID-G-RAA-ESSALUD-10), la autora procedió a la recolección de datos durante el periodo enero-marzo del 2011; inicialmente se accedió a las historias clínicas de los pacientes para decidir su condición de participante o no. Luego se realizaron las visitas domiciliarias para levantar la información sobre la variable en estudio, previo consentimiento informado del paciente y familiar responsable; la entrevista fue realizada con pacientes diagnosticados de cáncer de diversas etiologías en etapa terminal, todos asistentes a la Unidad de Terapia del Dolor y Cuidados Paliativos del área de estudio; siendo que la entrevista tuvo una duración aproximada de 15 a 20 minutos.

Para el análisis de la información, se elaboró una base de datos en el programa Excel 2007, utilizando códigos para preservar el anonimato de los pacientes. En relación a la variable en estudio, se usó el número cero (0) para las respuestas afirmativas y uno (1) para las negativas. Información que fue transportada al paquete estadístico SPSS versión 12, para su procesamiento estadístico respectivo, haciendo uso de la estadística descriptiva y para medir el nivel de calidad de vida se utilizó la prueba de estaninos, la cual fue determinada como: Alta (0-10), Media (11-14) y Baja (15-34).

\section{RESULTADOS}

El análisis descriptivo indica que la muestra fue conformada por 24 mujeres $(47,1 \%)$ y 27 hombres $(52,9 \%)$, destacando que el 33,3\% (17) se encontraba entre 80 y 88 años de edad. La mayor proporción de pacientes tenía primaria completa (51\%/26), donde el 47,1\% (24) estaba casado y $47,1 \%$ (24) jubilado. De la población en estudio, el $45,1 \%$ (23) vivía con esposa(o) e hijos, siendo que el $94,1 \%$ (48) tenía hijos y el $98 \%$ (50) profesaba la religión católica.

En relación a la localización de la neoplasia, el 23,5\% (12) presentó cáncer de próstata, el $17,6 \%$ (9) de mama, el 9,8\% (5) en cérvix, mientras que otros $(15,7 \%)$ estuvieron en ganglios, vejiga, leucemia, endometrio, óseo, recto y útero. De las neoplasias descritas, las que presentaron metástasis en mayor proporción fueron el cáncer de mama y el de próstata $(13,7 \% / 7)$ y cáncer de cérvix $(5,8 \% / 3)$. A seguir se muestran los gráficos que describen la calidad de vida de los pacientes.

Gráfico 1: Clasificación de la calidad de vida del paciente terminal según dimensión funcional, Unidad de Terapia del Dolor y Cuidados Paliativos/HNGAI, marzo 2011.

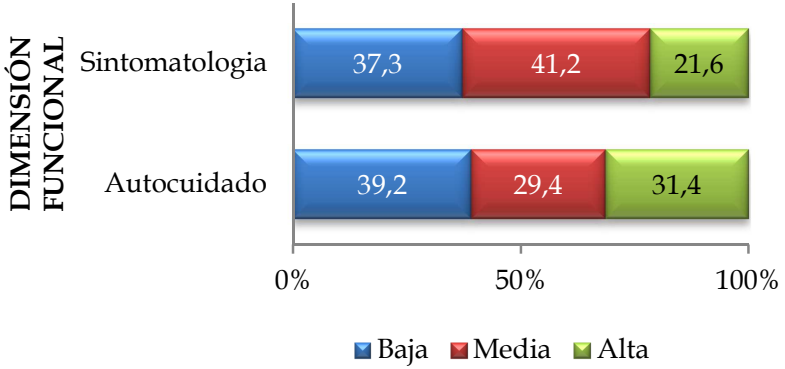


El gráfico 1 muestra que del 100\% (51) de entrevistados, por indicadores, el 41,2\% (21) presentó una calidad de vida media en "sintomatología", específicamente porque todos los pacientes manifestaron dolor intenso y debilidad para realizar su actividades diarias; otros datos importantes en este indicador fueron la pérdida de apetito $(98 \% / 50)$, pérdida de peso $(96,1 \% / 49)$, boca seca $(88,2 \% / 45)$ y dificultad para dormir $(84,3 \% / 43)$, además de estreñimiento, dificultad para respirar, náuseas y vómitos en menor frecuencia.

En relación al indicador "autocuidado", el $39,2 \%$ (20) de entrevistados presentó una calidad de vida baja, en la que el 68,6\% (35) manifestó ser dependiente en sus actividades diarias como: alimentación, higiene, vestimenta y otros. En general, en datos no mostrados se evidencia que el $60,8 \%$ (31) de entrevistados presentó una calidad de vida media en la dimensión funcional, con tendencia a una calidad de vida baja $(27,5 \%)$.

Gráfico 2: Clasificación de la calidad de vida del paciente terminal según dimensión psicológica, Unidad de Terapia del Dolor y Cuidados Paliativos/HNGAI, marzo 2011.

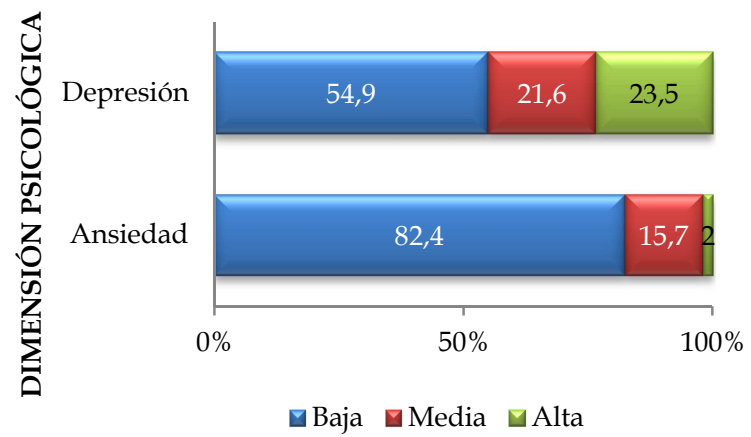

En el gráfico 2, se observa por indicadores que el $82,4 \%$ (42) de entrevistados presentó una calidad de vida baja por predominio de "ansiedad", destacando que el $98 \%$ (50) refirió sentirse tensionado/nervioso y preocupado $\mathrm{y}$, un $84,3 \%$ (43) manifestaba sentirse irritable. En relación a la calidad de vida baja para el indicador "depresión" que representa el 54,9\% (28), dentro de ellos el $72,5 \%$ (37) manifestó haber perdido el interés por las cosas y la confianza en sí mismo y el $64,7 \%$ (33) presentó desesperanza en la dimensión psicológica. En general, en datos no mostrados se aprecia que el $47,1 \%$ (24) de entrevistados presentó una calidad de vida baja en la dimensión psicológica y apenas el 9,8\% (5) una calidad de vida alta, observándose predominio de una calidad de vida media-baja.

Gráfico 3: Clasificación de la calidad de vida del paciente terminal en su dimensión social, Unidad de Terapia del Dolor y Cuidados Paliativos del HNGAI, marzo 2011.

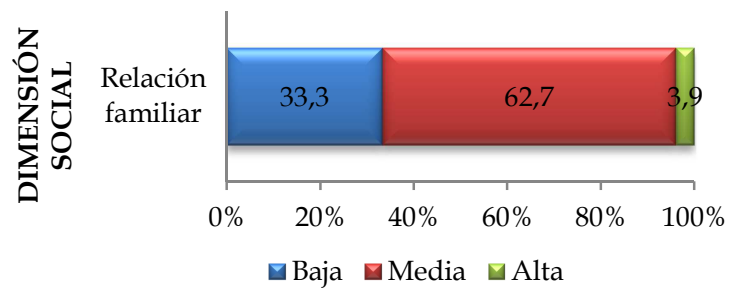

El gráfico 3 muestra que el 62,7\% (32) de los entrevistados presentó una calidad de vida media y $33,3 \%$ (17) baja en la dimensión social, evidenciándose una "relación familiar" disfuncional, especialmente porque el paciente considera que es una carga para la familia, de quienes no recibe apoyo emocional, estando insatisfecho con el tiempo que le dedican, siente además que no le escuchan y que el profesional de enfermería no apoya suficientemente a su familia para saber cuidarlo.

Gráfico 4: Clasificación de la calidad de vida del paciente terminal según dimensión espiritual, Unidad de Terapia del Dolor y Cuidados Paliativos del HNGAI, marzo 2011.

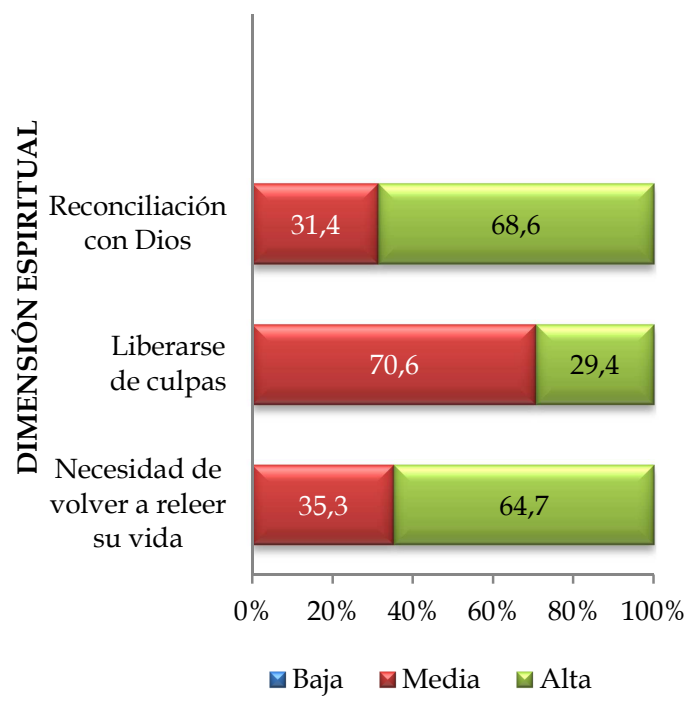


El gráfico 4 muestra en el indicador "reconciliación con Dios" que el 68,6\% (35) de entrevistados presentó una calidad de vida alta, principalmente porque todos respondieron que sienten necesidad de recibir los sacramentos y de confesarse, otro $88,2 \%$ (45) siente necesidad de reconciliarse con Dios y el 78,4\% (40) que la enfermedad pone a prueba su fe. En relación al indicador "liberarse de culpas" el 70,6\% (36) presentó una calidad de vida media, destacándose que el 74,5\% (38) sentía la necesidad de perdonar algún tema con su familia, amigos u otros. En el indicador "necesidad de volver a releer la vida", el 64,7\% (33) presentó una calidad de vida alta, siendo que al $66,7 \%$ (33) le gustaría que se le reconozca las mejores cosas que ha hecho en su vida y el 96,1\% (49) daría mayor importancia a cosas que antes no daba en su vida. En general, en datos no mostrados se evidencia que el $88,3 \%$ presentó una calidad de vida de media a alta (37,3\% y $51,0 \%$ respectivamente) en la dimensión espiritual.

Gráfico 5: Clasificación de la calidad de vida del paciente terminal en la Unidad de Terapia del Dolor y Cuidados Paliativos del HNGAI, marzo 2011.

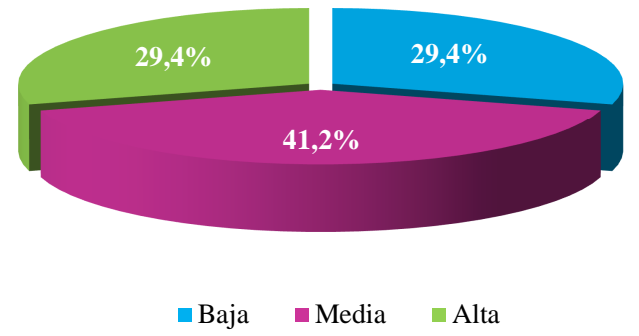

Gráfico 6: Clasificación de la Escala de Karnofski del paciente terminal, Unidad de Terapia del Dolor y Cuidados Paliativos del HNGAI, marzo 2011.

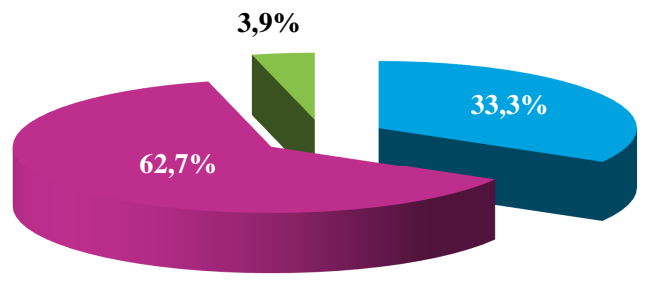

Incapacitado $\quad$ Afectado Normal
En el gráfico 5 se evidencia que el 41,2\% (21) de entrevistados presentó, en general, una calidad de vida media a partir las tres dimensiones establecidas para el estudio. Información que se complementa con los datos del gráfico 6, el cual muestra que el $62,7 \%$ (32) tenía afectado su capacidad para desarrollar actividades físicas.

\section{DISCUSIÓN}

En las últimas décadas, con las nuevas tecnologías médicas y el advenimiento de fármacos modernos, se ha prolongado la supervivencia de la población junto con su envejecimiento progresivo, observándose aumento en la prevalencia de enfermedades crónico degenerativas, entre ellas el cáncer, que derivan lentamente en una fase avanzada y terminal, con toda una serie de problemas físicos, emocionales, sociales y espirituales; donde la muerte es inevitable. $^{8}$

El cáncer, desde su inicio, provoca frecuentemente muchos síntomas y éstos se hacen cada vez más cambiantes y complejos según avanza la enfermedad, afectando la calidad de vida de la persona. Muchos autores afirman que la persona con cáncer, sobre todo, en fase avanzada y terminal, es un enfermo plurisintomático y multicambiante. ${ }^{9} \quad$ Un estudio realizado por Twycross, citado por Redondo, ${ }^{10}$ demostró que los enfermos terminales tenían entre 3 y 18 síntomas, con un promedio de 10 síntomas por enfermo, entre los síntomas más incidentes se encontraban el dolor, estreñimiento, debilidad, insomnio, anorexia, boca seca, disnea, ansiedad, náuseas y vómitos, depresión y problemas familiares.

Lo y Cols, citados por Peñacoba et al., ${ }^{7}$ refieren que una dimensión fundamental en la calidad de vida del paciente terminal es el aspecto físico; siendo la sintomatología uno de los principales aspectos a tener en cuenta. En el estudio de Taboada, ${ }^{11}$ cerca del $90 \%$ de enfermos terminales presentó dolor intenso, $60 \%$ dificultad para respirar $y$, casi todos, falta de apetito, sequedad bucal, constipación, debilidad general u otras molestias similares a los resultados obtenidos en el estudio, pues el $100 \%$ de los pacientes presentó dolor intenso y debilidad, seguido de falta de apetito $(98 \%)$, pérdida de peso $(96,1 \%)$, boca seca $(88,2 \%)$, dificultad para dormir $(84,3 \%)$ y otros como estreñimiento, dificultad para respirar, náuseas y vómitos (gráfico 1). Datos que coinciden con el estudio de Espantoso et al., ${ }^{12}$ aunque en menor frecuencia como dolor intenso y persistente 
$(39,6 \%)$, cansancio $(37,7 \%)$, alteraciones del sueño $(32,1 \%)$, pérdida de apetito y caquexia $(18,9 \%)$.

Gonzáles et al. ${ }^{13}$ acotan que el dolor es el síntoma más esperado y temido por los pacientes, así como uno de los más frecuentes. Se presenta entre un $30-40 \%$ de pacientes en la fase inicial de la enfermedad, que aumenta hasta el 70-90\% cuando la enfermedad está en estado avanzado. Entre aquellos que solicitan eutanasia activa, el $56 \%$ lo hizo por un sentimiento de pérdida de dignidad y $47 \%$ abogando tener un dolor intenso intratable. ${ }^{11}$

Vinaccia et al. ${ }^{14}$ refieren que el dolor afecta todas las dimensiones de la calidad de vida del paciente; existiendo una relación directa entre la calidad de vida e intensidad del dolor, aflicción que definitivamente le impide llevar una vida tranquila. Los autores arriba citados informan que alrededor del $69 \%$ de los pacientes con dolor crónico consideran que esa aflicción influenció mucho en su calidad de vida; contrastando con los resultados obtenidos en el estudio, en el que predominó la calidad de vida de media-baja (gráfico 1), reconociéndose al dolor como el síntoma de "excelencia" en todo los pacientes terminales. Fase en que, aliviar el dolor se convierte en imprescindible para ayudar a manejar los momentos finales de la vida; utilizando para esto diversos mecanismos alternativos, además de los fármacos de uso regular, como la escucha activa, la compañía, entre otros. ${ }^{15}$

En lo referente al indicador autocuidado, el $68,6 \%$ de los pacientes en estudio presentó dependencia en su cuidado personal (gráfico 1), información que se corrobora con la evaluación física realizada a través de la escala de Karnofski (gráfico 6). Datos que difieren del estudio de Peñacoba et al., ${ }^{7}$ quienes encontraron que los pacientes se consideran independientes en sus actividades de autocuidado, con niveles elevados de autosuficiencia $(63,0 \%)$. No obstante, se destaca que cada paciente y cada grupo de pacientes en condición terminal no pueden ser generalizados al colectivo de pacientes, más aun considerando los criterios de selección propuestos por los autores en referencia. Entre tanto, Carreras y Fernández ${ }^{16}$ revelan que el grado de satisfacción del paciente en relación a sus cuidados personales está acorde con la sintomatología que van apareciendo conforme progresa la enfermedad.

En la dimensión psicológica de la calidad de vida, Montoya ${ }^{17}$ hace referencia a los trastornos de ansiedad y depresión que son comunes en enfermedades graves, como el cáncer; Taboada ${ }^{11}$ refiere que el $80 \%$ de enfermos terminales presenta depresión o ansiedad. En el estudio se observa que los pacientes entrevistados en fase terminal, presentaban síntomas de depresión y ansiedad, las cuáles influyen decididamente en la determinación de la calidad de vida: depresión en el $54,9 \%$ de pacientes y ansiedad en el $82,4 \%$ (gráfico 2). En contraste, Suárez et al. ${ }^{18}$ identificaron que la ansiedad afectó al $28,2 \%$ de pacientes y la depresión al 23,1\%; ambas patologías suelen ser muy comunes entre los pacientes con cáncer.

White y Macleod ${ }^{19}$ mencionan que la depresión es más frecuente en la etapa terminal del cáncer, especialmente entre aquellos con síntomas físicos pobremente controlados; siendo que puede ser hasta tres veces más frecuente en los pacientes al final de la vida, que en la población general. ${ }^{20}$

La asociación de la ansiedad y el cáncer se relaciona con el miedo a la enfermedad, muerte o tratamiento, lo que provoca la aparición de síntomas físicos y psicológicos que afectan a la persona en su calidad de vida y en la forma de afrontar la enfermedad. Comúnmente una persona que experimenta ansiedad se queja de sentirse nerviosa, tensa, aprehensiva e irritable. ${ }^{21}$ Datos que se confirman en el estudio, donde el $98 \%$ de los pacientes respondió sentirse tensionado o nervioso $\mathrm{y}$ preocupado, mientras que un $84,3 \%$ se encontraba irritable.

La presencia de la enfermedad terminal en un miembro de la familia supone un fuerte impacto emocional para ésta y el enfermo. Condición que supone una profunda crisis en el funcionamiento de la familia, ${ }^{22}$ reconociéndose que la familia es el pilar para proveer cuidados al paciente terminal, especialmente para brindar apoyo afectivo y emocional.

En el estudio de Vinaccia et al., ${ }^{15}$ se resalta que el apoyo social que recibían los pacientes, expresado en la relación familiar, en general fue percibido como pobre. Dato que se asemeja con los resultados del estudio, en el que predominó una calidad de vida de media-baja (gráfico 3), identificándose la existencia de una relación familiar disfuncional. Peñacoba et al. ${ }^{11}$ relatan que ocho de cada 10 pacientes sentían que su enfermedad había afectado su relación familiar. De ellos, una proporción importante de pacientes consideró que el grado de afectación había sido alto y muy alto $(45,5 \%$ y $31,8 \%$ respectivamente). 
Copperman, citado por Toledo et al., ${ }^{23}$ al analizar los temores más frecuentes que viven las familias de pacientes con cáncer que mantienen una relación familiar disfuncional identificaron que sienten mucho miedo porque no saben cómo cuidar al paciente y no saben qué decir ante el sufrimiento del ser querido. Situación que los induce a recurrir a la espiritualidad y religión en busca de ayuda y soporte; otros optan por abordar estos temas con sus cuidadores. En un estudio, el $77 \%$ de pacientes deseaba que el profesional de salud tuviese en cuenta sus necesidades espirituales, otros $37 \%$ preferían que sus médicos les hablasen con más frecuencia sobre estas necesidades y $48 \%$ desearían que les acompañasen en la oración. ${ }^{24}$

Según Mejía, el aspecto espiritual de la vida humana puede verse como un componente integrado junto con los elementos físico, psicológico y social. Pero, para los que están cercanos al final de la vida, se asocia comúnmente con la necesidad de perdón, de reconciliación y de afirmación de los valores. $^{24}$

En el estudio, el 70,6\% de los entrevistados tuvo la necesidad de liberarse de culpas, otros expresaron también la necesidad de reconciliarse con Dios y otros la necesidad de volver a releer sus vidas, pudiendo experimentar estos sentimientos simultáneamente (gráfico 4). Un estudio sobre la evaluación de las necesidades espirituales en pacientes diagnosticados de cáncer avanzado y terminal, valorados en una escala de 0-10 puntos, reportó ellos tienen la necesidad de volver a releer la vida (6,2 puntos); necesidad de liberarse de la culpabilidad, de perdonar y necesidad de reconciliación (1,5 puntos) y, de sentirse perdonados (1,4 puntos). ${ }^{25}$

En el indicador liberarse de culpas, el 74,5\% de los entrevistados tuvo la necesidad de perdonar algún tema pendiente con su familia, amigos y otros; mientras que el 45,9 (23/51) sintió que su enfermedad era un castigo de Dios. Contrastando con los resultados de Vilalta, ${ }^{25}$ ya que la mayoría de los pacientes estudiados no tuvo la necesidad de perdonar nada, ni creyeron que la enfermedad era un castigo de Dios.

En el estudio, el $100 \%$ de pacientes tuvo necesidad de recibir los sacramentos y confesarse con el sacerdote, pastor y/o consejero espiritual, otro $88,2 \%$ sintió necesidad de reconciliarse con Dios y recibir el perdón de otra persona (familia, amigos y/o conocidos), reconociendo además que la enfermedad ponía a prueba su fe $(78,4 \%)$. Al respecto, Barbero ${ }^{20}$ identificó que el $24 \%$ de participantes percibió que recibir los sacramentos puede ayudarle a una continuidad, trascendencia y reconciliación con Dios y $46 \%$ refirió que siempre se pone a prueba la fe. Para Maté ${ }^{26}$ la fe se pone a prueba en la enfermedad grave, por el sufrimiento y la aproximación de la muerte.

Cuanto a la Necesidad de volver a releer la vida, el 96,1\% manifestó dar mayor importancia a cosas que antes no daba. Dato contrarios al reportado por Vilalta, ${ }^{25}$ donde el $68 \%$ de pacientes frecuentemente tuvo necesidad de organizar una nueva jerarquía de valores, otorgaron mayor importancia a cosas que antes no daban.

En general, todos los aspectos analizados hasta aquí, condicionan y determinan la calidad de vida, siendo que en el estudio los pacientes presentaron en mayor proporción una calidad de vida media (gráfico 5). Carreras y González ${ }^{16}$ encontraron en sus estudios que apenas el 4,5\% de pacientes tenía una buena o aceptable calidad de vida y $76,1 \%$ presentó una calidad de vida objetivamente pobre, coincidiendo con los resultados de Monge, $^{27}$ donde el $81,3 \%$ de población clasificó su calidad de vida como mala.

La calidad de vida es entendida como la sensación personal de bienestar que procede de la satisfacción o insatisfacción en las áreas de la vida que son importantes para la persona, generalmente están vinculados a determinadas dimensiones (físico, psicológico, conductual y otros). ${ }^{25}$

Un aspecto relevante que puede condicionar mucho la sensación de la calidad de vida, es el estatus funcional del paciente, referido a su capacidad de ejecutar una gama de actividades que son normales para la mayoría de personas, destacándose aquí la actividad física. En el estudio el $62,7 \%$ de los entrevistados presentó una actividad física afectada y un 33,3\% estaba en condición de incapacidad (gráfico 6). Contrarios a los de Carreras y González, ${ }^{16}$ dónde el 67,8\% de pacientes estaba incapacitado y requería de asistencia médica.

La importancia del estudio radica en la ausencia de investigaciones de este tema en el país y que los trabajos realizados en el extranjero muestran resultados variables, con escasa capacidad de generalización. Siendo muy pocos los estudios que describan o evalúen la calidad de vida del paciente oncológico en fase terminal. 
Frente a esta problemática, la enfermera es la profesional del equipo de salud, que brinda cuidado y da respuesta a las necesidades del paciente con el único fin de poder lograr una muerte digna en los pacientes terminales, defendiendo sus derechos, respetando su autonomía y dignidad, acompañándolo para mitigar su sufrimiento y lograr bienestar espiritual en el paciente y familia.

\section{Correspondencia:}

Jackelyn Cecilia Salas Pardo

Correo electrónico: jsalaspardo@ @otmail.com

\section{REFERENCIAS BIBLIOGRÁFICAS}

1. International Agency for Research on Cancer. Latest world cancer statistics Global cancer burden risesto 14.1 million new cases in 2012: Marked increase in breast cancers must be addressed [sede web]. Lyon/Geneva: WHO; 2013 [citado jul 30 de 2014]. Disponible en: http://www.iarc.fr/en/mediacentre/pr/2013/pdfs/pr223_E.pdf

2. Sepúlveda C. Nueva guía sobre cuidados paliativos de personas que viven con cáncer avanzado [sede web]. Ginebra/Suiza: OMS; 2007 [citado 30 jul de 2014]. Disponible en: http://www.who.int/mediacentre/news/notes/ 2007/np31/es/

3. Marco Mundial de Vigilancia Integral, con Inclusión de Indicadores y un Conjunto de Objetivos Mundiales de Aplicación Voluntaria para Prevenir y Controlar las Enfermedades No Transmisibles [sede web]. WHO; 2012 [citado 30 jul de 2014]. Disponible en: http://www.who.int/nmh/events/2012/Discuss ion_paper3 ES.pdf

4. Acedo F. Noticias de Salud Blogs [internet]. Los expertos demandan que la estrategia contra el cáncer una la investigación básica y clínica. 2008 Octubre- [acceso 02 de julio de 2014]. Disponible en: http://noticiadesalud.blogspot.com/2008/10/1 os-expertos-demandan-que-la-estrategia.html

5. Análisis de la Situación del Cáncer en el Perú 2013 [sede web]. Lima/Perú: MINSA; 2013 [acceso 30 de julio de 2014]. Disponible en: http://www.dge.gob.pe/portal/docs/asis_canc er.pdf

6. Huerta E. Cuida tu salud Blogs [internet]. El Dr. Ebert Poquioma y su registro de cáncer. 2008 Agosto- [citado 30 feb de 2011]. Disponible
Entre las limitaciones que pueden identificarse en el estudio, se destacan los relacionados con su diseño y el muestreo no probabilístico, siendo necesario replicar el mismo con poblaciones de gran envergadura y también con enfoques de naturaleza cualitativa, a fin de conocer las vivencias y sentimientos de este grupo humano.

http://elcomercio.pe/blog/cuidatusalud/2008/ $\underline{\text { 08/historia-del-dr-ebert-poquioma }}$

7. Peñacoba PC, Fernández SA, Morato CV, González Gutiérrez JL, López López A, Moreno Rodríguez R. Una aproximación a la calidad de vida de los enfermos ingresados en Unidades de Cuidados Paliativos. 2004, 14 (1):13-23

8. Consejo General de Colegios Oficiales de Psicólogos. Psicología, Cáncer y Cuidados Paliativos. INFOCOP [internet] 2006 marzoabril [citado 5 feb de 2011]; (27). Disponible en: http://www.cop.es/infocop/pdf/1101.pdf

9. Gómez SM. Control de síntomas: Incidencia y Principios generales del control de síntomas. Sistemas de evaluación. En: Gómez Sancho M. (ed). Cuidados Paliativos e Intervención Psicosocial en Enfermos Terminales. Las Palmas de Gran Canaria: ICEPSS, 1994:41-48

10. Redondo RB, Chacón RM, Grau AJA, Nicot Verdecia L. Evaluación de la sintomatología más frecuente en el paciente oncológico en fase terminal. Rev. Cubana Oncol [internet]. 1998 [citado 5 feb de 2011]; 14 (2): 83-6. Disponible en: http://bvs.sld.cu/revistas/onc/vol14_2_98/onc 02298.htm

11. Taboada RP. El derecho a morir con dignidad. Acta bioeth [internet]. 2000 [citado 5 feb de 2011]; 6 (1): 89-101. Disponible en: http://www.scielo.cl/pdf/abioeth/v6n1/art07.p df

12. Espantoso R, Fernández $\mathrm{C}$, Padierna $\mathrm{C}$, Amigo I, Villoria E, Gracia JM, et al. Calidad de vida en pacientes oncológicos un año después de finalizado el tratamiento. 
Psicooncologia [internet]. 2007 [citado 5 feb de 2011]; 4 (1): 43-57. Disponible en: http://revistas.ucm.es/index.php/PSIC/article/ viewFile/PSIC0707120043A/15727

13. Gonzáles A, Fernández C, García G, Amigo I, Arce C, Padierna $\mathrm{C}$ et al. Control de síntomas en pacientes oncológicos terminales ingresados en hospitalización domiciliaria. Psicooncología [internet]. 2005 [citado $5 \mathrm{feb}$ de 2011]; 2 (1): 3-20. Disponible en: http://revistas.ucm.es/index.php/PSIC/article/ view/PSIC0505120003A/16076

14. Vinaccia S, Quiceno JM, Fernández $\mathrm{H}$, Contreras F, Bedoya M, Tobón S, et al. Calidad de vida, personalidad resistente $\mathrm{y}$ apoyo social percibido en pacientes con diagnóstico de Cáncer pulmonar. Psicología y Salud [internet]. 2005 [citado 5 feb de 2011]; 15 (2):207-220. Disponible en: http://revistas.uv.mx/index.php/psicysalud/art icle/view/805

15. Astudillo W, Mendinueta C, Orbegozo A. Presente y futuro de los Cuidados Paliativos [internet]. SN: Paliativos sin fronteras.com [citado jul 20 del 2014]. Disponible en: http://www.paliativossinfronteras.com/upload /publica/cuidados\%20paliativos/documentos/ PRESENTE\%20Y\%20FUTURO\%20CUIDA DOS\%20PALIATIVOS.pdf

16. Carreras RO, Gonzáles PJR. Calidad de vida al egreso en pacientes con Cáncer Paliativo y terminal. MEDISAN [internet]. 2002 [citado 30 jul de 2014]; 6 (2): 6-11. Disponible en: http://www.bvs.sld.cu/revistas/san/vol16_2_0 2/san02202.pdf

17. Montoya D. Importancia del soporte psicosocial para los pacientes con cáncer. Boletín de Psicología [internet]. Dic 1999 [citado 25 mar de 2011]; 1 (1). Disponible en: http://www.humanas.unal.edu.co/nuevo/inde x.php

18. Suárez A, Ávila P, Caballero D, Cuca L. síntomas de ansiedad y depresión en un grupo de pacientes oncológicos del Hospital Departamental de Villavicencio [Tesis de licenciatura]. Colombia: Universidad El Bosque; 2005. Disponible en: http://www.uelbosque.edu.co/sites/default/fil es/publicaciones/facultades/psicologia/rais_2 0-enero-2014.pdf

19. White CA, Macleod U. Cáncer. BMJ [internet]. 2002 [citado 5 feb de 2011]; 325 (7360): 377-380. Disponible en:
http://www.ncbi.nlm.nih.gov/pmc/articles/P MC1123889/

20. Barbero J. Psicólogos en Cuidados Paliativos: La sinrazón de un olvido. Psico Oncología [internet] 2008 [citado 27 mar de 2011]; 5 (1): 179-191. Disponible en: http://revistas.ucm.es/index.php/PSIC/article/ view/PSIC0808130179A/15553

21. Velásquez MN, Vázquez TLG, Alvarado AS. Ansiedad asociada al dolor agudo en pacientes oncológicos. Cancerología [internet]. 2009 [citado 27 mar de 2011]; 4: 19-29. Disponible en: http://www.incan.org.mx/revistaincan/elemen tos/documentosPortada/1257541120.pdf

22. Muñoz CF, Espinosa AJM, Portillo SJ, Rodríguez GMG. La familia en la enfermedad terminal I. Medicina de Familia (And) [internet]. Sep 2002 [citado 27 mar de 2011]; 3 (3): 190-199. Disponible en: http://www.samfyc.es/Revista/PDF/v3n3/07. pdf

23. Toledo M, Barreto MP, Sánchez-Cánovas J, Martínez E, Ferrero J. La enfermedad terminal: La muerte y los cuidados paliativos. 2001; 13 (2): 5-47.

24. Mejía BA. Aspectos psico-emocionales y espirituales al final de la vida: El proceso de morir dignamente. Medicina Naturista [internet]. 2008 [citado 4 mar de 2011]; 2 (3): 233-245. Disponible en: http://dialnet.unirioja.es/servlet/articulo?codi go=2666025

25. Vilalta A. Evaluación de las necesidades espirituales de pacientes diagnosticados de cáncer avanzado y terminal [Tesis Doctoral]. España: Universidad de Leida; 2010. 219p. Disponible en: http://www.tdx.cat/bitstream/handle/10803/8 072/Tavv1de1.pdf;jsessionid=02BB861E3A1 D69A7CBEA53E4AF79F1F9.tdx2?sequence $=1$

26. Maté EJJ. ¿Qué dicen los expertos sobre las necesidades espirituales?: Revisión bibliográfica. En: Benito E, Barbero J, Payás A. ed. El acompañamiento espiritual en cuidados paliativos: Una introducción y una propuesta [internet]. Madrid: SECPAL; 2008 [citado 20 jul de 2014]. Disponible en: http://books.google.com.pe/books?id=2W8M $\underline{61 \mathrm{zQHg} 8 \mathrm{C} \& p g=\mathrm{PA} 57 \& \operatorname{lpg}=\mathrm{PA} 57 \& \mathrm{dq}=\% \mathrm{C} 2}$ $\% \mathrm{BFQu} \% \mathrm{C} 3 \% \mathrm{~A} 9+$ dicen+los+expertos+sobr e+las+necesidades+espirituales?:+Revisi\%C3 \%B3n+bibliogr\%C3\%A1fica.\&source=bl\&ot 
$\underline{\mathrm{S}=-}$

R83tZbN3D\&sig=FCkylePOKmTR4WBc4t5 cUjSJwqg\&hl=es\&sa=X\&ei=X01RVKKwB 8SfgwTwqIPIAg\&ved=0CBoQ6AEwAA\#v= onepage\&q=\%C2\%BFQu $\% \mathrm{C} 3 \% \mathrm{~A} 9 \% 20$ dice n\%201os\%20expertos\%20sobre\%20las\%20n ecesidades\%20espirituales\%3F\%3A\%20Revi si\%C3\%B3n\%20bibliogr\%C3\%A1fica.\&f=fa $\underline{\text { lse }}$

27. Monge RFS. Calidad de vida en pacientes con cáncer de mama - Hospital EsSalud Cusco. Situa [internet]. 2006 [citado 30 jul de 2014]; 15(1,2): 48-54. Disponible en: http://sisbib.unmsm.edu.pe/bvrevistas/situa/2 006_n1-2/pdf/a09.pdf 